\title{
Potentiale sektorübergreifender Innovationen am Beispiel der Schlaganfallversorgung
}

STEPHAN VON BANDEMER, JOSEF HILBERT,

ANNA NIMAKO-DOFFOUR

Stephan von Bandemer ist Koordinator des Arbeitsbereichs Versorgungsforschung, Innovationsmanagement und Internationalisierung im Forschungsschwerpunkt Gesundheitswirtschaft und Lebensqualität des Instituts Arbeit und Technik in Gelsenkirchen

Prof. Dr. Josef Hilbert ist Geschäftsführender Direktor und Direktor des Forschungsschwerpunktes Gesundheitswirtschaft und Lebensqualität des Instituts Arbeit und Technik in Gelsenkirchen

Anna Nimako-Doffour ist wissenschaftliche Mitarbeiterin im Arbeitsbereich Versorgungsforschung, Innovationsmanagement und Internationalisierung im Forschungsschwerpunkt Gesundheitswirtschaft und Lebensqualität des Instituts Arbeit und Technik in Gelsenkirchen

\author{
Schlaganfallversorgung erfolgt notwendigerweise \\ sektorübergreifend. Während die Akutversorgung in \\ Stroke Units hoch standardisiert und gut organisiert \\ ist, fehlen in der prä- und postakuten Phase der \\ Versorgung entsprechende Standards, so dass erhebliche \\ Versorgungsunterschiede entstehen. Eine Abstimmung \\ von Versorgungsstandards ist mit Selektivverträgen \\ allerdings nicht zu erreichen, sondern erfordert \\ kollektivrechtliche, korporatistische Lösungen.
}

\section{Burdon of Desease und Integrierte Versorgung}

In deutschen Krankenhäusern wurden 2012 insgesamt 409.758 Schlaganfälle behandelt (ICD G45, I60-I64) (1). Schlaganfälle waren die fünfthäufigste Todesursache in Deutschland (2). International gelten sie als einer der häufigsten Ursachen für Behinderungen im Erwachsenenalter (3). Die Kosten für die Versorgung von Schlaganfallpatienten für deren gesamte Lebensdauer wurden bereits für das Basisjahr 2004 mit 50.507 Euro kalkuliert (4) und dürften mittlerweile deutlich höher liegen. Schlaganfälle bilden damit sowohl unter gesundheitlichen Gesichtspunkten als auch unter Kostenaspekten eine der größten Herausforderungen der Gesundheitsversorgung.

Chancen wie Grenzen sektorübergreifender Versorgung lassen sich am Beispiel des Schlaganfalls gut analysieren, da hier alle Sektoren, Dienstleister, die medizintechnische Industrie, aber auch Akutversorgung ebenso wie Primär- und Sekundärprävention an der Versorgung beteiligt werden müssen. Neben vorbildlichen Versorgungsstrukturen, die auch international als best practice angesehen werden, sind in Deutschland in der Schlaganfallversorgung auch erhebliche Defizite festzustellen, deren Überwindung durch organisatorische wie technische Innovationen sowohl die Gesundheit verbessern als auch Kosteneinsparungen erreichen kann. Dabei spielt allerdings die Integrierte Versorgung mit den Gestaltungsmöglichkeiten des SGB V aus unterschiedlichen Gründen keine Rolle. Insofern kann die Schlaganfallversorgung auch als Beispiel dafür dienen, bestehenden Reformbedarf an der Regulierung der Integrierten Versorgung aufzuzeigen.

\section{Schlaganfallversorgung als integrierte Systemlösung}

Grundsätzlich erfordert die Schlaganfallversorgung sektorübergreifende Systemlösungen. Diese reichen von der Aufklärung der Öffentlichkeit über die Primärprävention, das Management des Rettungswesens, die Organisation von Stroke Units bis zur Rehabilitation, Sekundärprävention und Pflege (Abb. 1). Eine wirksame Versorgung funktioniert nur, wenn alle diese Bereiche systema- 
Abb.1: Versorgungskette Schlaganfall

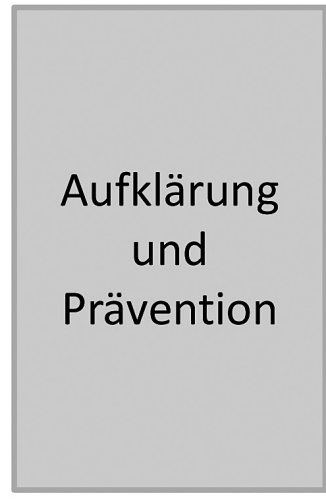

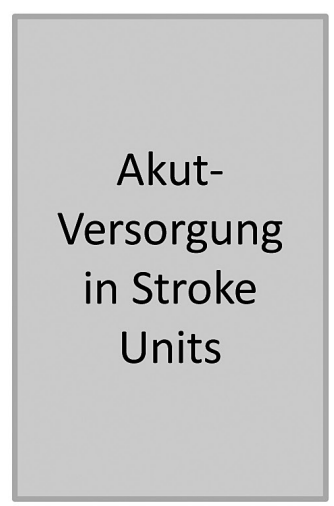

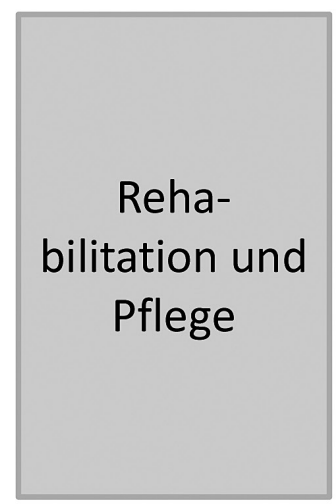

Sekundärprävention
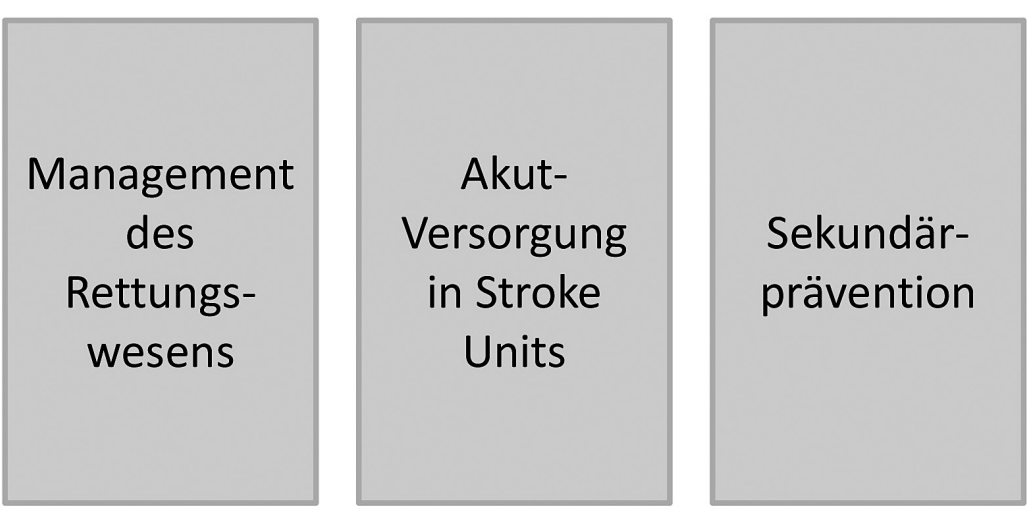

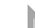

Quelle: JAT, Eigene Darstellung

tisch aufeinander abgestimmt sind und reibungslos ineinander greifen.

\subsection{Akutversorgung}

Einen der wichtigsten Bausteine der Akutversorgung des Schlaganfalls bilden Stroke Units, die in der Bundesrepublik seit 1996 systematisch auf- und ausgebaut wurden. Auf Initiative der wissenschaftlichen Fachgesellschaften (Deutsche Gesellschaft für Neurologie, Deutsche Schlaganfallgesellschaft, DSG) wurde 2005 eine Komplexpauschale für die Stroke Unit Behandlung eingeführt (5), die Grundlage für eine flächendeckende Verbreitung von Stroke Units wurde, die nach den Zertifizierungskriterien der DSG organisiert sind. Die Behandlung von Schlaganfallpatienten in den spezialisierten Stroke Units gilt mittlerweile auch international als Gold-Standard (6), und neben den Anforderungen in Deutschland wurden auch für Europa (7) oder die USA (8) ähnliche Anforderungen entwickelt.

Die Stroke Units verfolgen einen sektorübergreifenden Ansatz, allerdings ohne dabei auf die im SGB V verankerten Instrumentarien der Integrierten Versorgung zurückzugreifen. So erfolgt in der Akutphase notwendiger Weise eine enge Abstimmung mit den Rettungsdiensten, mit denen entsprechend der Zertifizierungskriterien Pathways abzustimmen sind, in denen die Schlaganfalldiagnostik, die Vorinformation der Stroke Units und die Übergaben bei der Aufnahme in die Stroke Unit zu regeln sind. In der
Post-akut Phase wird die Rehabilitation bereits in den Stroke Units begonnen und mit Rehabilitationsanbietern nach der Entlassung abgestimmt. Die Sekundärprävention wird ebenfalls bereits in der Stroke Unit eingeleitet. Die Finanzierung dieser Maßnahmen erfolgt dabei über eine OPS Komplexpauschale und nicht durch Integrierte Versorgungsverträge. Eine solche über das DRG System (und nicht kostenträgerspezifisch) geregelte Finanzierung ist allein schon deshalb erforderlich, weil es in der Akutversorgung der Schlaganfalls kaum vertretbar wäre, Art und Ausmaß der Behandlung am Versicherungsstatus der Patienten auszurichten. Hier sind für alle Patienten einheitliche Standards erforderlich, deren Finanzierung für alle Patienten gelten muss. Dies gilt unabhängig von der Diagnose für die Akutversorgung insgesamt.

Trotz des anspruchsvollen und einheitlichen Niveaus bei der Akutversorgung zeigen sich bereits beim Übergang in die Anschlussversorgung Probleme. Abgesehen von der Frührehabilitation der Phase A, die bereits innerhalb der ersten 24 Stunden nach Aufnahme der Patienten in der Stroke Unit beginnt, zeigen sich in den folgenden Rehabilitationsphasen große, vor allem regionale Unterschiede, die auf Sektor übergreifende Reibungsverluste und auf Steuerungssowie Entscheidungsprozesse mit fragmentierten Kompetenzen zurückgeführt werden müssen.

\subsection{Rehabilitation nach Schlaganfall}

Im Bereich der Rehabilitation ist insbesondere zwischen der Phase B (neurologische Frührehabilitation) und den Phasen $\mathrm{C}$ und $\mathrm{D}$ in stationären Rehabilitationseinrichtungen zu unterscheiden. Teilweise erfolgt auch eine Verlegung von Patienten aus den Stroke Units in die stationäre geriatrische Rehabilitation. Nationale und internationale Studien zeigen $(9,10,11)$,

Trotz des anspruchvollen und einheitlichen Niveaus bei der Akutversorgung des Schlaganfalls zeigen sich beim Übergang in die Anschlussversorgung Probleme.

dass sowohl die neurologische Frühreha als auch die Rehabilitation der Phase C und D zu erheblichen Verbesserungen des Gesundheitszustandes von Patienten nach Schlaganfall beitragen können. Hier bestehen jedoch jeweils gravierende Unterschiede in der Versorgung, die mit unterschiedlichen Steuerungsmechanismen und Finanzierungsformen zusammenhängen. Die Finanzierung erfolgt dabei sowohl über das DRG System (SGB V $\mathbb{S} 39$ ) als auch über die medizinische Rehabilitation (SGB V $\mathbb{4} 40$ ).

Bei der neurologischen Frühreha geht es um Patienten mit schweren und schwersten Hirnschädigungen (Barthel Index <30). In den meisten Bundesländern wird die neurologische Frühreha 
als Krankenhausbehandlung mit der OPS 8-552 („neurologisch-neurochirurgische Frührehabilitation") abgerechnet. Allerdings bestehen auch hier erhebliche Unterschiede im Angebot zwischen Bundesländern, die die neurologische Frühreha eher in spezialisierten Einrichtungen durchführen und anderen, die diese vorwiegend in Krankenhäusern mit intensivmedizinischer Versorgung anbieten (12). Zudem wird neurologische Frührehabilitation in einigen Rehakliniken angeboten, um möglichst fließende Übergänge zwischen der Phase $\mathrm{B}$ und $\mathrm{C}$ zu gewährleisten. Die unterschiedlichen Regelungen zur institutionellen Durchführung der neurologischen Frühreha und zur Abstimmung zwischen den Rehaphasen tragen mit dazu bei, dass regional große Schwankungen zwischen den Anteilen der Patienten bestehen, die eine neurologische Frühreha erhalten. Die Frührehaquote bei Schlaganfällen lag 2012

im Bundesdurchschnitt bei 9,02 Prozent mit einer Schwankung zwischen rund 16 Prozent (Thüringen 16,8, Bayern 15,37) und knapp 2 Prozent (Sachsen 1,87, NRW 1,94) (13).

Diese Unterschiede kommen u.a. dadurch zustande, dass die Landeskrankenhausplanung nicht verpflichtet ist, unterhalb der Fachdisziplinen Detailplanungen vorzunehmen. Während etwa in Bayern unterhalb der Landeskrankenhausplanung eine Abstimmung zwischen dem Gesundheitsministerium, den Kostenträgern und den Leistungserbringern erfolgte (14) und in Baden Württemberg eine eigenständige Fachplanung für die neurologische Frührehabilitation vorgenommen wurde (15), sind Krankenhäuser in anderen Bundesländern, etwa in NRW, auf die Budgetverhandlungen mit den Kostenträgern angewiesen. Diese werden dann aber von Kostenträgern zum Teil mit Verweis darauf, dass im Landeskrankenhausplan keine entsprechenden Betten ausgewiesen seien, gar nicht erst aufgenommen. Damit wird die neurologische Frühreha zur Einzelfallentscheidung mit erheblichen Unsicherheiten bei der Refinanzierung für die Krankenhäuser.

Auch in der Rehaphase C und D, die in Rehabilitationskliniken durchgeführt wird, bestehen große regionale Unterschiede. Der durchschnittliche Anteil der Schlaganfallpatienten, die eine stationäre Rehabilitation in Rehaeinrichtungen erhielt, lag 2009 im Bund bei 26,79 Prozent. Die Schwankungsbreite zwischen den Bundesländern lag dabei zwischen 11,72 Prozent in Hamburg und 36,55 Prozent in Sachsen. Die Schwankungsbreite auf Ebene der Kreise und kreisfreien Städte ist noch weit größer (16).

Insgesamt zeigen sich bei der Rehabilitation nach Schlaganfall sehr unterschiedliche Regelungen, die zu Problemen beim Übergang zwischen den Sektoren, zu großen Schwankungen bei der Verfügbarkeit der Rehabilitation für die Patienten und auch zu unterschiedlichen Finanzierungsmodellen führen. Integrierte Versorgungsstrukturen könnten hier zu erheblichen Verbesserungen führen, allerdings nur,

Integrierte

Versorgungsstrukturen können bei der Rehabilitation nach Schlaganfall zu erheblichen Verbesserung führen. Sie müssen jedoch flächendeckend angeboten werden.

wenn damit flächendeckende Angebote verbunden sind. Demgegenüber können Integrierte Versorgungsverträge mit einzelnen, vielfach regional aufgestellten Kostenträgern kaum zu einer Vereinheitlichung der Versorgung beitragen. Zwar ist der Wettbewerb zwischen den Kostenträgern vom Gesetzgeber intendiert, jedoch entsteht - zumindest bei der Schlaganfallversorgung - bei einer Abstimmung von Versorgungsstrategien mit einzelnen Kostenträgern ein unverhältnismäßig hoher Verwaltungsaufwand, der das Lösungspotenzial Integrierten Versorgung in seiner derzeitigen Form deutlich reduziert.

\subsection{Primär und Sekundärprävention}

Auch vor dem Hintergrund der epidemiologischen Daten und den mit Schlaganfällen verbundenen hohen
Kosten bestehen bei der Primär- und Sekundärprävention erhebliche Potenziale zur Reduzierung der Krankheitslast. Neben nicht beeinflussbaren Risikofaktoren für Schlaganfälle wie Alter und Geschlecht, bestehen eine Vielzahl von beeinflussbaren Faktoren wie Lebensstil (Rauchen, Bewegung) und Ko-Morbiditäten wie Bluthochdruck, Diabetes, Vorhofflimmern oder Hyperlipidämien. Insbesondere für die Sekundärprävention bestehen nationale und internationale Leitlinien $(17,18)$; dennoch sind etwa ein Viertel aller Schlaganfälle Reinsulte, die vielfach mit einer mangelnden Umsetzung therapeutischer Empfehlungen zusammenhängen (19).

Zwar beziehen sich die Leitlinien vorwiegend auf die Sekundärprävention, dennoch gelten vergleichbare Anforderungen an die Primärprävention (20, 21). Hier kommt insbesondere hinzu, dass eine Risikostratifizierung der $\mathrm{Pa}$ tienten erforderlich ist, um effiziente Präventionsstrategien zu entwickeln, deren Gestaltung aber komplementär zur Sekundärprävention ist. Dennoch werden viele Risikofaktoren nicht angemessen berücksichtigt bzw. therapiert. So bestehen vielfach Probleme bei modifizierbaren Risikofaktoren wie z.B. der Blutdruckeinstellung (22), den Blutzuckerwerten (23), der Antikoagulation (24) sowie Lebensstil Veränderungen (23), die dazu beitragen, dass die Zahl der Schlaganfälle kontinuierlich zunimmt.

Dabei bestehen - wie im Fall der Rehabilitation - erhebliche regionale Unterschiede. Während der bundesweite Durchschnitt der Hospitalisierungen bei ischämischen Schlaganfällen je 10.000 Einwohnern bei 29,6 Fällen liegt, liegt die Schwankungsbreite in den Kreisen und kreisfreien Städten zwischen 17,83 und 56,16 Fällen (25). Dies sagt zwar noch nichts über die Ursachen der Unterschiede aus, kann aber als Indiz dafür genommen werden, dass einerseits erhebliche Unterschiede in den Sozialstrukturen und Lebensstilen bestehen, andererseits aber vermutlich auch erhebliche Unterschiede der Versorgung zu verzeichnen sind. Unabhängig von den Ursachen zeigen die Daten, dass ein hoher Bedarf, aber auch realistische Möglichkeiten für die Umsetzung von Präventionsstrategien bestehen. 
Ansatzpunkte für Präventionsstrategien finden sich neben der Verhaltensprävention u.a. in systematischer Detektion und Behandlung von Risikofaktoren (im Bereich Primärprävention) sowie bei der Detektion der Ursachen kryptogener Schlaganfälle sowie der konsequenten medikamentösen Versorgung in der Sekundärprävention. Dies setzt Sektor und Fachgruppen übergreifend abgestimmte Strategien sowohl im Screening als auch bei der Medikation voraus und wäre grundsätzlich ein gu-

\section{Es sprechen gute Gründe für die Hypothese, dass Präventionsstrategien für Kostenträger unattraktiv sind, da Investitionen und Ertrag zeitlich deutlich auseinander fallen können.}

anfall in der Bundesrepublik sehr gut funktioniert, die Primärprävention und Anschlussversorgung aber erhebliche Defizite aufweisen.

\section{Barrieren und Potenziale integrierter Versorgung}

Das Beispiel der Schlaganfallversorgung zeigt, dass Integrierte Versorgungssysteme dringend benötigt werden. Viele Erst- und Re-Insulte ließen sich durch abgestimmte Präventionsstrategien verhindern und Pflegebedürftigkeit könnte durch abgestimmte Rehabilitationsleistungen reduziert werden. Allerdings hat sich das unter dem Dach des SGB V entstandene System der Integrierten Versorgung in der Bundesrepublik, auf das große Hoffnungen gesetzt wurde (30), bislang sehr zögerlich entwickelt und längst noch nicht durchgesetzt. Lediglich

ter Ansatzpunkt für umfassende und flächendeckend Integrierte Versorgung. Faktisch bestehen aber fast ausschließlich beispielhafte Strategien etwa im Rahmen von begrenzten Studien zur Sekundärprävention $(26,27)$ oder in Form von Registerstudien $(28 ; 29)$. Eine systematische Steuerung etwa im Rahmen integrierter Versorgungsansätze ist praktisch kaum zu verzeichnen.

Mögliche Ursachen für mangelnde Initiativen zur Integrierten Versorgung bei der Prävention von Schlaganfällen sind bislang nicht hinreichend erforscht. Allerdings sprechen gute Gründe für die Hypothese, dass Präventionsstrategien für Kostenträger unattraktiv sind, da Investitionen und Ertrag zeitlich deutlich auseinander fallen können, dass Investitionen und Ertrag sich auf unterschiedliche Budgets auswirken und deshalb für einzelne Akteure unattraktiv sind, dass Komplexität und Aufwand für einzelne Kostenträger und einzelne Leistungserbringer zu hoch sind und eher kollektive Steuerung erfordern und dass die Verantwortungsteilung etwa zwischen der Sozialpolitik im Bereich der Verhaltensprävention und der Gesundheitspolitik im Bereich der Bereitstellung notwendiger medizinischer Leistungen in der Prävention ungeklärt ist. Insofern ist zu erklären, dass die Akutversorgung beim Schlag-
0,7 Prozent der Gesundheitsausgaben fließen in die Integrierte Versorgung und davon entfallen allein 44 Prozent auf die Knappschaft mit einer entsprechenden Sonderstellung (31). Dieser Widerspruch spricht dafür, dass der Integrierten Versorgung in der Bundesrepublik Konstruktionsfehler zugrunde liegen.

Ein zentraler Problembereich der Integrierten Versorgung scheint zu sein, dass hiermit Wettbewerbsanreize zwischen den Kostenträgern verbunden werden. Damit ist die Wirkung der Integrierten Versorgung im Akutbereich praktisch ausgeschlossen, da Patienten im medizinischen Notfall nicht erst nach ihrem

Versichertenstatus gefragt werden dürfen. In der Schlaganfallversorgung zahlt es sich hier aus, dass durch die Einführung der DRG Komplexpauschale für die Akutversorgung eine gute finanzielle Grundlage geschaffen wurde, die auch sektorübergreifende Konzepte zumindest in Ansätzen zulässt.

Ein zweiter Problembereich zeigt sich am Beispiel der Rehabilitation. Eine umfassend und flächendeckend Integrierte Versorgung kann, wie das Beispiel Bayern zeigt, entweder im Rahmen einer Mehrebenenpolitik und -steuerung, an der die Landeskrankenhausplanung, die Kostenträger, Krankenhäuser und Rehabilitationseinrichtungen beteiligt sind, korporatistisch, d.h. in wechselseitiger Abstimmung, angegangen werden. Oder sie gelingt dann, wenn Kostenträger und Leistungserbringer - wie im Fall der Knappschaft - in einem Boot sitzen („Bottroper Modell“,32). Beide Fälle haben aber nichts mit wettbewerblichen Ansätzen zu tun, wie sie bislang im SGB V im Mittelpunkt stehen.

Das Beispiel der Primär- und Sekundärprävention zeigt Probleme der Integrierten Versorgung, wenn es zu Inkongruenz der Planungszeiträume oder der betroffenen Budget Verantwortlichkeit kommt. Investitionen in die Prävention, die sich erst mittelfristig refinanzieren, führen zu hoher Unsicherheit und damit in der Tendenz zur Innovationszurückhaltung. Dies gilt umso mehr, wenn die Investitionsbudgets zumindest nur zum Teil vom return on investment profitieren (Investition der Krankenversicherung führt zu Einsparungen in der Pflegeversicherung).

Wenn das deutsche Gesundheitssystem von den Chancen der Integrierten Versorgung profitieren will, muss es sich daher vermutlich von der Idee der Steuerung über Wettbewerb verabschieden und Lösungen anbieten, bei denen sekto-

Wenn das deutsche Gesundheitssystem von den Chancen der Integrierten Versorgung profitieren will, muss es sich vermutlich von der Wettbewerbssteuerung verabschieden.

rübergreifende Angebote in die Regelversorgung integriert werden. Einheitliche Versorgungsstandards werden sich nur durch eine Abkehr von Selektivverträgen und eine stärkere Nutzung von sektorübergreifenden Kollektivverträgen erreichen lassen. Hierfür gilt es Modelle zu entwickeln und zu erproben, die z.B. indikationsbezogen sektorübergreifende Budgets bereitstellen oder zumindest sektorübergreifend Lösungen verbindlich vorschreiben. 


\section{Literatur}

(1) Statistisches Bundesamt: DRG Statistik 2012

(2) Statistisches Bundesamt: Ergebnisse der Todesursachenstatistik für Deutschland - Ausführliche vierstellige ICD1O-Klassifikation - 2012

(3) Johnston SC, Mendis S, Mathers CD. Global variation in stroke burden and mortality: estimates from monitoring, surveillance, and modelling. Lancet Neurol 2009; 8: 345-354.

(4) Peter L. Kolominsky-Rabas, Peter U. Heuschmann, Daniela Marschall, Martin Emmert, Nikoline Baltzer, Bernhard Neundörfer, Oliver Schöffski and Karl J. Krobot: Lifetime Cost of Ischemic Stroke in Germany: Results and National Projections From aPopulation-Based Stroke Registry: The Erlangen Stroke Project. Stroke. 2006; 37:1179-1183

(5) E. Bernd Ringelstein und Otto Busse: Stroke Units in Deutschland - Gefährdung eines Erfolgsrezeptes? GGW 3/2004 (Juli), 4. Jg.

(6) Stroke Unit Trialists' Collaboration. Organised inpatient (stroke unit) care for stroke. Cochrane Database of Systematic Reviews 2013, Issue 9. Art. No.: CDoo0197.

(7) E. Bernd Ringelstein, Angel Chamorro, Markku Kaste, Peter Langhorne, Didier Leys, Philippe Lyrer, Vincent Thijs, Lars Thomassen and Danilo Toni for the ESO Stroke Unit Certification Committee: European Stroke Organisation Recommendations to Establish a Stroke Unit and Stroke Center. Stroke. 2013; 44:828-840.

(8) Mark J. Alberts, Richard E. Latchaw, Andy Jagoda, Lawrence R. Wechsler, Todd Crocco, Mary G. George, E.S. Connolly, Barbara Mancini, Stephen Prudhomme, Daryl Gress, Mary E. Jensen, Robert Bass, Robert Ruff, Kathy Foell, Rocco A. Armonda, Marian Emr, Margo Warren, Jim Baranski, Michael D. Walker and for the Brain Attack Coalition: Revised and Updated Recommendations for the Establishment of Primary Stroke Centers: A Summary Statement From the Brain Attack Coalition. Stroke. 2011;42:2651-2665

\section{(9) Turner-Stokes L, Nair A, Sedki I,Disler} PB,Wade DT.Multi-disciplinary rehabilitation for acquired brain injury in adults of working age. Cochrane Database of Systematic Reviews 2005, Issue 3. Art.No.: CDo04170.

(10) Geschäftsstelle Qualitätssicherung Hessen: Schlaganfallbehandlung Neurologische Rehabilitation. Jahresauswertung 2011.

(11) Leitlinien der Deutschen Gesellschaft für Neurologie: Leitlinien für Diagnostik und Therapie in der Neurologie; 4. überarbeitete Auflage 2008, S. $654 \mathrm{ff}$, ISBN.

(12) Stefan Loos, Anke Schliwen: Gutachten zur Abbildung der neurologisch-neurochirurgischen Frührehabilitation im Rahmen der Krankenhausplanung. Endbericht für die Landesarbeitsgemeinschaft NeuroRehabilitation NRW, Berlin 2012
(13) Statistisches Bundesamt: DRG Statistik 2012

(14) M. Schorl, D. Liebold: Neurologisch-neurochirurgische Frührehabilitation in Deutschland - Aktuelle Situation, Probleme und Lösungsmöglichkeiten aus medizinischer und juristischer Sicht. Akt Neurol 2012; 39: 1-11.

(15) Ministerium Für Arbeit und Sozialordnung, Familie, Frauen und Senioren Baden-Württemberg, Neurologische Frührehabilitation Phase B. Fachplanung für BadenWürttemberg, 2012.

(16) Statistische Landesämter, Rehabilitationsstatistik, 2009.

(17) Hans-Christoph Diener, Christian Weimar, Die neue S3 Leitlinie "Schlaganfallprävention“ der Deutschen Gesellschaft für Neurologie und der Deutschen Schlaganfallgesellschaft. Psychopharmakotherapie, 2013; 20: 58-65

(18) Karen L. Furie, Scott E. Kasner, Robert J. Adams, Gregory W. Albers, Ruth L. Bush, Susan C. Fagan, Jonathan L. Halperin, S. Claiborne Johnston, Irene Katzan, Walter N. Kernan, Pamela H. Mitchell, Bruce Ovbiagele, Yuko Y. Palesch, Ralph L. Sacco, Lee H. Schwamm, Sylvia Wassertheil-Smoller, Tanya N. Turan and Deidre Wentworth on behalf of the American Heart Association Stroke Council, Council on Cardiovascular Nursing, Council on Clinical Cardiology, and Interdisciplinary Council on Quality of Care and Outcomes Research: Guidelines for the Prevention of Stroke in Patients With Stroke or Transient Ischemic Attack: A Guideline for Healthcare Professionals From the American Heart Association/American Stroke Association. Stroke 2011:42:227-276

(19) K. Schneider, M. Heise, P. Heuschmann, K. Berger: Lebens- und Versorgungssituation von Schlaganfallpatienten. 3-Monats-Follow-up des Qualitätssicherungsprojektes Nordwestdeutschland. Nervenheilkunde 3/2009: 114-118.

(20) Martin J O'Donnell, Denis Xavier, Lisheng Liu, Hongye Zhang, Siu Lim Chin, Purnima Rao-Melacini, Sumathy Rangarajan, Shofi qul Islam, Prem Pais, Matthew J McQueen, Charles Mondo, Albertino Damasceno, Patricio Lopez-Jaramillo, Graeme J Hankey, Antonio L Dans, Khalid Yusoff, Thomas Truelsen, Hans-Christoph Diener, Ralph L Sacco, Danuta Ryglewicz, Anna Czlonkowska, Christian Weimar, Xingyu Wang, Salim Yusuf, on behalf of the INTERSTROKE investigators:

Risk factors for ischaemic and intracerebral haemorrhagic stroke in 22 countries (the INTERSTROKE study): a case-control study. Lancet 2010: 376; 112-23.

(21) E. Bernd Ringelstein, Henning Henningsen: Primärprävention des Schlaganfalls. Deutsches Ärzteblatt Jg. 98, Heft 6, 9. Februar 2001

(22) Amytis Towfighi, Daniela Markovic, Bruce Ovbiagele: Consistency of Blood Pressure Con- trol After Ischemic Stroke. Prevalence and Prognosis. Stroke. published online March 27, 2014

(23) P. Ott, I. Benke, J. Stelzer, C. Vöhler, M.Hanefeld: Diabetes in Germany (DIG)-Studie. Prospektive 4-Jahresstudie zur Therapiequalität bei Typ-2-Diabetes in der Praxis. Dtsch Med Wochenschr 2009; 134: 291-297.

(24) Jonas Oldgren, Jeff S. Healey; Michael Ezekowitz; Patrick Commerford; Alvaro Avezum; Prem Pais; Jun Zhu; Petr Jansky; Alben Sigamani; Carlos A. Morillo; Lisheng Liu; Albertino Damasceno; Alex J. Grinvalds; Juliet Nakamya; Paul A. Reilly; Katalin Keltai; Isabelle C. Van Gelder; Afzal Hussein Yusufali; Eiichi Watanabe; Lars Wallentin; Stuart J. Connolly; Salim Yusuf: Variations in Etiology and Management of Atrial Fibrillation in a Prospective Registry of 15,400 Emergency Department Patients in 46 Countries: The RE-LY AF Registry. Circulation. Published online before print January 24, 2014

(25) Statistisches Bundesamt 2012: DRG Statistik.

(26) Timolaos Rizos, Janina Güntner, Ekkehart Jenetzky, Lars Marquardt, Christine Reichardt, Rüdiger Becker, Roland Reinhardt, Thomas Hepp, Paulus Kirchhof, Elena Aleynichenko, Peter Ringleb, Werner Hacke and Roland Veltkamp: Continuous Stroke Unit Electrocardiographic Monitoring Versus 24Hour Holter Electrocardiography for Detection of Paroxysmal Atrial Fibrillation After Stroke. Stroke. 2012;43:2689-2694.

(27) International Stroke Conference (ISC) 2014. Abstract LB11

(28) Karsten Keller: Quality of oral anticoagulation in venous thromboembolism - results from the thrombEVAL trial, 22.2.2013. 57. Jahrestagung der Gesellschaft für Thrombose- und Hämostaseforschung (GTH), 20. bis 23. Februar 2013, München. Session 06: Venous Thrombosis.

(29) VHF-Schlaganfallregister, Nordwestdeutsche Stroke Units (interne Daten).

(30) Rürup B, IGES Institut, DIW Berlin e.V., DIW econ GmbH, Wille E. (2010) Effizientere und leistungsfähigere Gesundheitsversorgung als Beitrag für eine tragfähige Finanzpolitik in Deutschland, Baden-Baden.

(31) Sachverständigenrat zur Begutachtung der Entwicklung im Gesundheitswesen (2012): Wettbewerb an der Schnittstelle zwischen ambulanter und stationärer Gesundheitsversorgung. Sondergutachten.

(32) Georg Greve, Hans Adolf Müller, Stefan Hörter. „Prosper-Modelle der KnappschaftStrukturen, Finanzierungsaspekte und Prozessmanagement in der Integrierten Versorgung der Knappschaft Bahn See." In: Günther E. Braun: Innovative Versorgungsformen im Gesundheitswesen: Konzepte und Praxisbeispiele erfolgreicher Finanzierung und Vergütung (2009): 75 